\title{
Biomechanics of the heel pad for type 2 diabetic patients
}

\author{
Tsz-Ching Hsu ${ }^{\text {a }}$, Ying-Shiung Lee ${ }^{\mathrm{b}}$, Yio-Wha Shau ${ }^{\mathrm{c}, *}$ \\ ${ }^{a}$ Department of Rehabilitation Medicine, Chang Gung Memorial Hospital, Tao-Yuan, Taiwan, ROC \\ ${ }^{\mathrm{b}}$ Laboratory of Electron Microscope, Department of Cardiology, Chang Gung Memorial Hospital, Tao-Yuan, Taiwan, ROC \\ ${ }^{\mathrm{c}}$ Institute of Applied Mechanics, National Taiwan University, No. 1, Roosevelt Rd., Sec. 4, Taipei 106, Taiwan, ROC
}

Received 10 August 2001; accepted 8 March 2002

\begin{abstract}
Objectives. To quantify the dynamic behavior of the heel pad in type 2 diabetic patients and age-matched healthy individuals using mathematical modeling.

Background. No single parameter can fully describe the heel-pad biomechanical properties during the loading-unloading process.

Design. A descriptive study using pseudoelastic modeling was conducted to simulate the heel-pad stress-strain relationship in the loaded and unloaded states. Transmission electron microscope was used to examine six heel specimens taken from amputated legs in diabetic and non-diabetic patients.

Methods. Energy dissipation ratio, loading curvature, and unloading curvature were calculated from the stress-strain curve-fits. Differences in ultrastructure between the heel pad of healthy subjects and those with diabetes were described.

Results. The diabetic patients had a significantly higher mean energy dissipation ratio (mean $36.1 \%$ (SD, $8.7 \%$ ) vs mean $27.9 \%$ $(\mathrm{SD}, 6.1 \%) ; P<0.001)$ and mean unloaded curvatures (mean $11.8(\mathrm{SD}, 5.1)$ vs mean $8.46(\mathrm{SD}, 2.6) ; P<0.001)$ than those of the control group. The collagen fibrils in diabetic heel samples were ruptured with unclear striation and uneven distribution.

Conclusions. The curvature parameters may explain the poor rebound phenomenon resulting in the high impact energy in diabetic heel pads. Breakdown in collagen fibrils may be responsible for this observation.
\end{abstract}

\section{Relevance}

These findings can be integrated into the fabrication of orthotics that dissipate excessive heel impact energy and protect against injury. (c) 2002 Elsevier Science Ltd. All rights reserved.

Keywords: Heel; Biomechanics; Diabetes foot; Modeling; Collagen

\section{Introduction}

The heel pad, located beneath the calcaneus bone, acts as an efficient shock absorber to reduce potential injury to the body during ambulation [1]. Diabetic foot ulcers, the end stage of a series of harmful cascades that initiated by hyperglycemia, are caused by multiple pathologies [2], in which the altered foot mechanical properties resulting from the changes in fibrous structure may contribute in part to the development of foot ulceration [3].

Several methods, involving a universal material testing machine [4], the drop impact test [5], the ballistic pendulum [6], the roentgenography [7], and the durometer [8] have been used to investigate the heel-pad

\footnotetext{
${ }^{*}$ Corresponding author.

E-mail address: ywshau@spring.iam.ntu.edu.tw (Y.-W. Shau).
}

mechanical properties in vivo or in vitro. We built a loading-unloading apparatus that incorporated an ultrasound transducer with a force gauge to measure the dynamic heel-pad properties at different conditions [911]. This system enabled the change in the heel-pad thickness during compression to be visualized directly. Higher impact energy was found to dissipate in the diabetic heel pad than those of the age-matched healthy individual [9]. However, there was no significant difference in the tissue stiffness between the two groups. Therefore, an improved understanding of the mechanical characteristics of the heel pad would be helpful for the prevention of further damage to the heel.

It is well known that the biological tissues are typically viscoelastic, and that hysteresis exists during the loading-unloading process. The energy dissipation ratio (EDR) had been proposed to quantify the viscoelastic behavior of the heel pad [9]. However, the changes in 
hysteresis may result from changes either in the loading phase or the unloading phase, or both. Thus the EDR alone is insufficient to describe the complex nature of the heel-pad viscoelasticity.

In this study, the pseudoelastic modeling was used to resolve the non-linear loading and unloading responses of the heel pad with two simple parameters respectively. The loading and unloading curves were treated separately as uniquely defined stress-strain relationship without temporal consideration [12]. The parameters derived from mathematical modeling of experimental data would provide a more systemic view of the complex biomechanical phenomenon [13].

We also performed the transmission electron microscopic examination for a few heel-pad specimens taken from the amputated feet to investigate the differences in collagen fibrils between non-diabetic individuals and diabetic patients, because the abnormal mechanical behaviors of the heel might be resulted from the changes in the internal soft-tissue structure. The purpose of this study was to determine the effect of changes in viscoelastic properties of the heel pad in type 2 diabetic patients and age-matched healthy individuals at both macroscopic and microscopic levels.

\section{Methods}

\subsection{Subjects}

Twenty age-matched healthy subjects (10 males and 10 females) and twenty-one patients (10 males and 11 females) with type 2 diabetes, fulfilling the diagnostic criteria of the American Diabetes Association [14], were recruited in the study. All subjects gave their informed consent before this non-invasive examination. The age, gender, and body mass index (BMI) in the two groups

Table 1

Demographic characteristics of subjects in different groups

\begin{tabular}{llll}
\hline & $\begin{array}{l}\text { Healthy } \\
\text { controls }\end{array}$ & $\begin{array}{l}\text { Diabetic } \\
\text { patients }\end{array}$ & $P$-value \\
\hline Sex (F/M) (n) & $10 / 10$ & $11 / 10$ & $0.986^{\mathrm{a}}$ \\
Age (mean, years) & 61.2 & 59.0 & $0.411^{\mathrm{b}}$ \\
& $(\mathrm{SD}, 7.3)$ & $(\mathrm{SD}, 9.8)$ & \\
& 23.7 & 23.7 & $0.963^{\mathrm{b}}$ \\
BMI (mean, $\left.\mathrm{kg} / \mathrm{m}^{2}\right)$ & $(\mathrm{SD}, 3.0)$ & $(\mathrm{SD}, 3.0)$ & \\
& 0 & 38 & $0.003^{\mathrm{c}}$ \\
Neuropathy $(\%)$ & & 9.75 & \\
Diabetes duration & & $(\mathrm{SD}, 8.0)$ & \\
(mean, years) & & 8.36 & \\
HbA $_{\text {lc }}(\%)$ & & $(\mathrm{SD}, 1.8)$ & \\
& &
\end{tabular}

Data is presented with mean (standard deviation) F: female; M: male; BMI: body mass index; $\mathrm{HbA}_{1 \mathrm{c}}$ : Glyohemoglobin.

${ }^{a}$ Pearson Chi-square test.

${ }^{\mathrm{b}}$ Student's $t$-test.

${ }^{\mathrm{c}}$ Fisher's exact test. were similar. All individuals had palpable pulse in their dorsalis pedis and posterior tibial arteries. No foot ulceration could be detected in the diabetic patients and their disease duration and Glycohemoglobin $\left(\mathrm{HbA}_{\mathrm{lc}}\right)$ level were recorded. The sensation of the mid-heel region was tested three times using the Semmes-Weinstein monofilament (North Coast Medical Inc., San Jose, CA, USA) of 5.07 (equivalent to 10 -g of linear force). Significant diabetic neuropathy was defined when patients were inability to sense this stimulation [15]. The diabetic patients had statistically higher incidence $(P=0.003)$ of neuropathy than that of the non-diabetic individuals. The clinical characteristics of all subjects are shown in Table 1 .

\subsection{Instrumentation}

A $10-\mathrm{MHz}$ linear-array ultrasound transducer (General Electric Company, LOGIQ 700MR, Milwaukee, Wisconsin, USA), with a contact area of $3.96 \mathrm{~cm}^{2}$, was incorporated into a push-pull scale (IMADA Inc., Hashira, Toyohashi, Japan) and then mounted on the self-constructed loading-unloading device. Both heels of all recruited individuals were examined. Each subject was placed in the prone position with ankle in neutral and knee in $90^{\circ}$-flexed positions. The skin was pretreated with alcohol to improve the transmission of ultrasound beam through the epidermis. We positioned the transducer along the line connecting the second toe and the mid-heel. Adequate amounts of jelly were applied between the ultrasound transducer and the plantar heel. The unloaded heel-pad thickness was measured just before the contact of transducer with the heel. In a loading-unloading cycle, the transducer gave the heel pad to the maximum contact stress of $74 \mathrm{kPa}$, resembling the peak standing in-shoe plantar contact stress measurement in the heel regions of healthy individuals [16], in increments of $12.4 \mathrm{kPa}$. The heel was then relaxed sequentially to the unloaded condition. Each measurement of the heel-pad thickness at certain contact stress took about $5 \mathrm{~s}$ and the transducer was moved manually at an average speed of about 0.0006 $\mathrm{m} / \mathrm{s}$ [9].

The strain was defined as the ratio of the heel-pad deformation to the unloaded thickness respectively. Beyond the maximum stress $\left(\sigma_{\max }=74 \mathrm{kPa}\right)$ typically, no further deformation was observed. The mean elastic modulus $\left(E_{\mathrm{p}}\right)$ given by the ratio of the maximum stress to the maximum strain was used as an alternative reference. The hysteresis, representing the dissipated energy in the heel pad, can be drawn from the bounded area of non-linear stress-strain curves. The EDR can be defined as (Fig. 1):

$\mathrm{EDR}=($ bounded area/area under loading curve $) \times 100 \%$ 


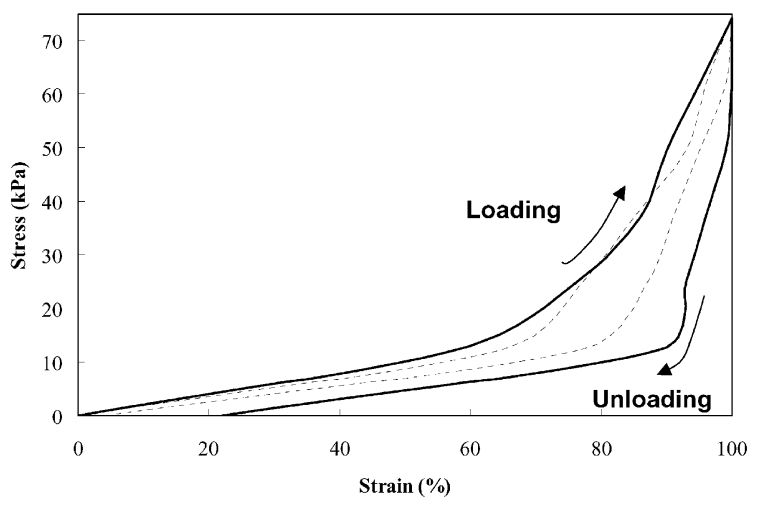

Fig. 1. Stress-strain curves for a 60-year-old healthy person (- - -), and a 58 -year-old type 2 diabetic patient $(-)$. The strain $(\varepsilon)$ here has been normalized by its maximum value for comparison of different subjects. $\varepsilon=$ heel-pad deformation/unloaded heel-pad thickness. The loading curve (upper) and the unloading curve (lower) form a closed area. EDR is defined as the ratio of the closed area to the area formed by the loading curve, the vertical dotted line, and the $\mathrm{X}$-axis.

\subsection{Biomechanical modeling}

Assume that the loading and unloading curves can be described separately using the following constitutive equation:

$\frac{\sigma_{\text {load }}}{\sigma_{\max }}=\left(\frac{\varepsilon}{\varepsilon_{\max }}\right)^{\alpha_{\text {load }}}$
$\frac{\sigma_{\text {unload }}}{\sigma_{\max }}=\left(\frac{\varepsilon}{\varepsilon_{\max }}\right)^{\alpha_{\text {unload }}}$

where $\sigma$ is the contact stress for the loading and unloading curves; $\sigma_{\max }$ the maximal contact stress, i.e. 74 $\mathrm{kPa}$, in this study, $\varepsilon$ the strain; $\varepsilon=$ (heel-pad deformation/unloaded heel-pad thickness); $\varepsilon_{\max }$ the maximal strain, $\alpha$ the curvature parameters for the loading and unloading curves. Therefore, the non-linear loading-unloading curve can be fully characterized using the four parameters, $\sigma_{\max }, \varepsilon_{\max }, \alpha_{\text {load }}$, and $\alpha_{\text {unload }}$.

\subsection{Ultrastructural studies}

Four diabetic and two non-diabetic heel specimens were taken from amputated feet due to severe leg trauma and forefoot gangrene respectively. The mean age of the four diabetic patients and the non-diabetic patients was $69.5(\mathrm{SD}, 6.4)$ years of age and $67.8(\mathrm{SD}, 7.7)$ years of age respectively. Tissues taken from the mid-heel region were firstly fixed with glutaraldehyde, then placed in solutions of buffered ice cold $1 \%$ osmium tetroxide and further stained with tannic acid. They were dehydrated with $100 \%$ ethanol at room temperature and defatted in a 50/50-chloroform/methanol solution for 3 days. After embedded in epon embedding medium and stained with $4 \%$ uranyl acetate and Reynold's lead citrate, these specimens were examined by Hitachii
H-7500 electron microscopy (Hitachii Co. Ltd., Tokyo, Japan) operated at $75 \mathrm{KV}$.

\subsection{Statistical analysis}

Student's $t$-test was used to analyze the age, BMI, EDR, loading curvature $\left(\alpha_{\mathrm{load}}\right)$, and unloading curvature $\left(\alpha_{\text {unload }}\right)$ between the diabetic and non-diabetic subjects. Pearson Chi-square test and Fisher's exact test were conducted to estimate the gender and the incidence of

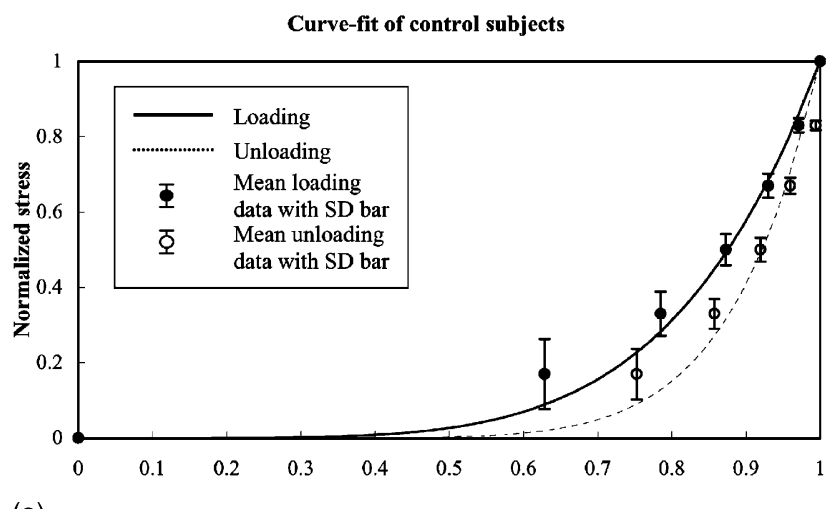

(a)

Normalized strain

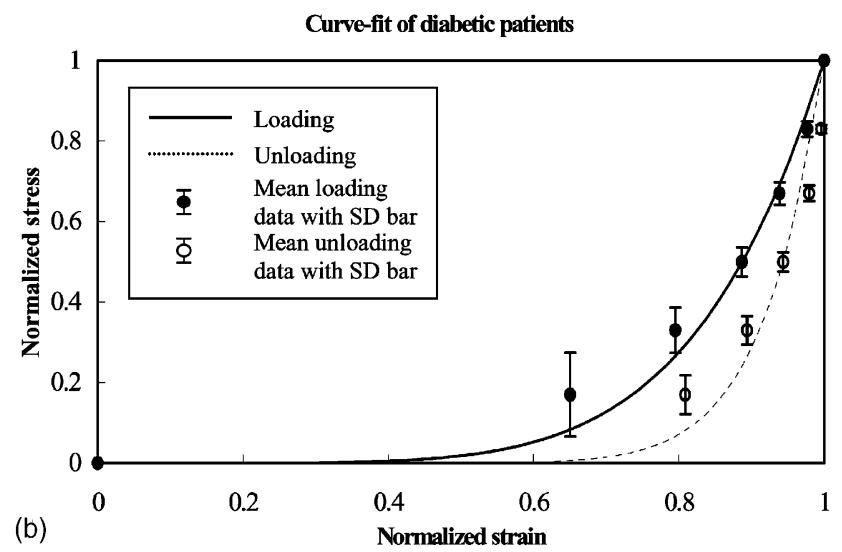

Fig. 2. Curve-fit for the heel-pad loading-unloading curves: (a) healthy control subjects; (b) diabetic patients. \$. mean normalized strain data in the fit loading curve (-) at certain stress with one standard deviation length bar; 호: mean normalized strain data in the fit unloading curve (- - ) with one standard deviation length bar.

Table 2

Mechanical properties of the heel pad in different groups (mean (SD))

\begin{tabular}{llll}
\hline & Group I & Group II & $P$-value \\
\hline EDR (\%) & $27.9(\mathrm{SD}, 6.1)$ & $36.1(\mathrm{SD}, 8.7)$ & $<0.001$ \\
$\alpha_{\text {load }}$ & $5.21(\mathrm{SD}, 1.5)$ & $5.77(\mathrm{SD}, 1.6)$ & 0.116 \\
$\alpha_{\text {unload }}$ & $8.46(\mathrm{SD}, 2.6)$ & $11.8(\mathrm{SD}, 5.1)$ & $<0.001$ \\
$\alpha_{\text {unload }}-\alpha_{\text {load }}$ & $3.25(\mathrm{SD}, 2.1)$ & $6.18(\mathrm{SD}, 4.5)$ & $<0.001$ \\
\hline
\end{tabular}

EDR: energy dissipation ratio; $\alpha_{\text {load }}$ : curvature of loading curves; $\alpha_{\text {unload }}$ : curvature of unloading curves; Group I: healthy subjects; Group II: type 2 diabetic patients.

${ }^{\text {a }}$ Student's $t$-test. 
neuropathy respectively between the two groups. Correlation between EDR, $\alpha_{\text {load }}, \alpha_{\text {unload }}$, and $\left(\alpha_{\text {unload }}-\alpha_{\text {load }}\right)$ was also evaluated. A $P$-value of $<0.05$ was regarded as of statistical significance.

\section{Results}

Fig. 2 demonstrated the curve-fit results of loading and unloading curves using pseudoelastic model. The
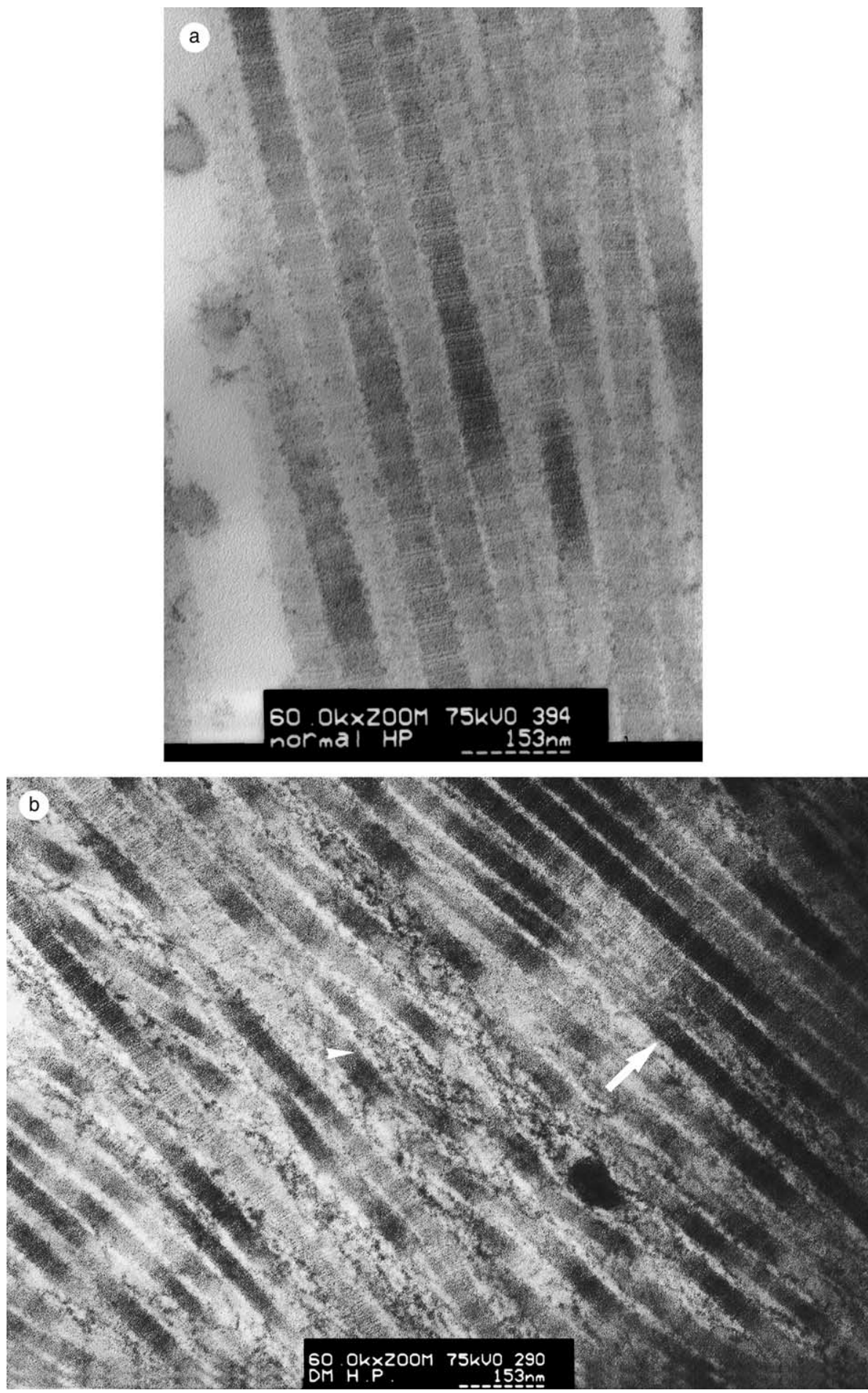

Fig. 3. (A) Longitudinal views of the collagen fibrils within septal walls of the heel pad of a 65 -year-old non-diabetic individual. Collagen fibrils appear in parallel and demonstrate typical band periodicity (magnification 60,000×). (B) Longitudinal views of the collagen fibrils within septal walls of the heel pad of a 68-year-old diabetic patient. The right upper corner shows relatively normal collagen fibrils (arrow). Most of the field has fragmented and distorted collagen fibrils. They were irregularly distributed in the area (magnification $60,000 \times$ ) with thin filament (arrowhead). 
diabetic patients had significantly $(P<0.001)$ higher energy dissipated in their heel pads, greater mean $\alpha_{\text {unload }}$ value and greater mean $\left(\alpha_{\text {unload }}-\alpha_{\text {load }}\right)$ value than those of non-diabetic persons (Table 2). The difference in the mean loading curvature between non-diabetic individuals was not significant. Fair correlation between EDR and $\alpha_{\text {unload }}$ was observed. However, a higher correlation was found between the EDR and $\left(\alpha_{\text {unload }}-\alpha_{\text {load }}\right)$. The information was summarized in the Table 2 .

The longitudinal appearance of the collagen fibrils within septal walls in non-diabetic heel tissue appeared paralleled arranged. Typically, the averaged band periodicity was about $60 \mathrm{~nm}$ and a clear interfibrillar space could also be identified (Fig. 3A). The collagen fibrils were not evenly involved in diabetic heel pads. Most of the fibrils appeared distorted and fragmented. The band periodicity disappeared and thin filamentous strands distributed in a disorganized manner (Fig. 3B).

\section{Discussion}

Modeling, the attempt to represent the reality may provide more insight into the true nature of the system of interest and can estimate or predict variables of interest [13]. A non-linear theory of the Kelvin type has been proposed to depict living tissues behavior on the basis of a sequence of springs of different natural length, with the number of participating springs increasing with increasing strain. The hysteresis curves, a salient feature of most biological soft tissue, can be derived from such a simulation [17]. Although collagen tissue may be responsible for this phenomenon, only qualitative account of how the septa may be distorted when the cushion is loaded [3]. To date, no quantitative investigation of the heel-pad micromechanics, the way in which the components interact to deliver the observed mechanical properties, has been published [18].

The heel-pad mechanical properties had been studied using the CI, the $E_{\mathrm{p}}$, and the EDR in different population [4-9,11]. The CI and $E_{\mathrm{p}}$ were determined simply from the unloaded and end-loaded conditions. These quantities along cannot present the non-linear stressstrain characteristics of the heel pad. The impact energy dissipated in the soft tissues may be important in quantifying viscoelasticity. However, how the stressstrain curvatures or non-linearity change in the diabetic patients required new thoughts.

In this study, the pseudoelastic model provided a simple analysis tool to quantify the behavior of stressstrain curve. Fig. 2 showed the typical curve-fit results for the heel pad in an age-matched healthy subject. The model function gives a good representation for the loading-unloading paths respectively. There was no statistical difference in the $\alpha_{\text {load }}$ between the two groups of subjects in our study while the diabetic patients had a higher $\alpha_{\text {unload }}$ than that of the non-diabetic subjects. It is believed that the increased unloading curvature, resulting in an increase of the difference between the unloading and loading curvatures, is the major cause for increased EDR in the heel pad. Although poor rebound phenomena have been observed in diabetic patient [9], aging individual [10], and the patient with reconstructed musculocutaneous flap [11], no quantitative index has been given. The curvatures of the loading and unloading curves may serve as additional parameters to represent the heel-pad mechanical properties.

The light microscopic examination for diabetic heel pads revealed the distorted and ruptured fibrous tissue strands and atrophic fat globules [19]. In addition to the previous reports, our study demonstrated that the collagen fibrils in diabetic heel pads were involved in varied areas at varied extents. The typical band periodicity of each involved collagen fibrils disappeared. The deleterious effects of glycation, a non-enzymatic reaction, on collagen play central roles in the evolution of disorganized collagen fibrils [20]. Advanced glycation end products are formed and lead to intermolecular crosslinks between the collagen molecules. These links may drastically change the charge profiles and reduce the uptake of heavy metal anion on collagen fibrils. These effects may further cause the typical band periodicity to disappear in diabetic patients [21]. Glycation does not have specific trend and occurs at different rates within the collagen unit cell [22]. It is conceived that the collagen fibrils are not evenly affected. These qualitative changes of collagen fibrils may influence the tissue properties of the heel pad and finally lead to the development of foot ulceration.

The heel pad of healthy elderly adults had higher dissipated energy than that of the healthy young subject. This finding may be responsible for the higher incidence of heel injury in elderly individuals [8]. While comparing with uninjured heel pads, elevated EDR could also be demonstrated in injured heel pads reconstructed with musculocutaneous flaps, which may lead to the development of shock-induced discomfort and ulceration [11]. Perhaps an increased dissipated energy damages the heel-pad and increase the susceptibility to foot ulceration in diabetic patients. These observations may result from disorganized collagen fibrils, which make the occurrence of poor rebound phenomenon, especially in diabetic patients.

The intrinsic heel-pad tissue viscoelasticity can be better described using a simple pseudoelastic modeling. The mathematical analysis offers a more systemic approach to the heel-pad biomechanics. The alteration of the heel-pad mechanical properties in the diabetic patients may be related to the breakdown of collagen fibrils. The curvature parameters provided by mathematical modeling may be useful for evaluating the underlying pathology of diabetic heel pads and bridging 
the gap between the podiatric science and the proper design of foot orthotics. Moreover, the shear stress may also be important in the pathogenesis of the heel problems. Therefore, further investigation is indicated.

\section{References}

[1] Sarrafian SK. Functional anatomy of the foot and ankle. In: Sarrafian SK, editor. Anatomy of the foot and ankle, descriptive, topographic, functional, 2nd ed. Philadelphia: J.B. Lippincott; 1993. p. 474-602.

[2] Cevera JJ, Bolton LL, Kerstein MD. Options for diabetic patients with chronic heel ulcers. J Diab Comp 1997;11:358-66.

[3] Jahss MH, Michelson JD, Desai P, Kaye R, Kummer F, Buschman W, et al. Investigations into the fat pads of the sole of the foot: anatomy and histology. Foot Ankle Int 1992;13:233-42.

[4] Bennet MB, Ker RF. The mechanical properties of the human subcalcaneal fat pad in compression. J Anat 1990;171:131-8.

[5] Kinoshita H, Francis PR, Murase T, Kawai S, Ogawa T. The mechanical properties of the heel pad in elderly adults. Eur J Appl Physiol 1996;43:404-9.

[6] Aerts P, Ker RF, De Clerzq D, Ilsley SW. The effects of isolation on the mechanics of the human heel pad. J Anat 1996;188:417-23.

[7] Prichasuk PM, Mulpruek P, Siriwongpairat P. The heel-pad compressibility. Clin Orthop Relat Res 1994;300:197-200.

[8] Brink T. Induration of the diabetic foot pad: another risk factor for recurrent neuropathic plantar ulcers. Biomedizinische Technik 1995;40:205-9.

[9] Hsu TC, Wang CL, Shau YW, Tang FT, Li KL, Chen CY. Altered heel-pad mechanical properties in type 2 diabetic patients. Diabetic Med 2000;17:854-9.

[10] Hsu TC, Wang CL, Tsai WC, Kuo JK, Tang FT. Comparison of the mechanical properties of the heel pad between young and elderly adults. Arch Phys Med Rehabil 1998;79:1101-4.
[11] Wang CL, Shau YW, Hsu TC, Chen HC, Chien SH. Mechanical properties of heel pads reconstructed with flaps. J Bone Joint Surg [Br] 1999;81-B:207-11.

[12] Fung YC. Bioviscoelastic solids. In: Fung YC, editor. Biomechanics: mechanical properties of living tissues, 2nd ed. New York: Springer-Verlag; 1993. p. 242-320.

[13] Nigg BM. Modelling. In: Nigg BM, Herzog W, editors. Biomechanics of the musculoskeletal system, 2nd ed. Chichester: John Wiley \& Sons; 1999. p. 423-634.

[14] American Diabetes Association. Report of the Expert Committee on the diagnosis and classification of diabetes mellitus. Diabetes Care 1997;20:1183-97.

[15] Laing N. Diabetic foot ulcers. Am J Surg 1994;167(suppl. $1 \mathrm{~A}): 31 \mathrm{~S}-6 \mathrm{~S}$.

[16] Rozema A, Ulbrecht JS, Pamme SE, Cavanagh PR. In-shoe plantar pressures during activities of daily living: implications for therapeutic footwear design. Foot Ankle Int 1996;17: $352-9$.

[17] Viidik A. Biomechanics and functional adaptation of tendons and joint ligaments. In: Evans FG, editor. Studies on the anatomy and function of bone and joints. New York: Springer-Verlag; 1966. p. $17-39$.

[18] Ker RF. The design of soft collagenous load-bearing tissues. J Exp Biol 1999;202:3315-24.

[19] Buschmann WR, Jahss MH, Kummer F, Desai P, Gee RO, Ricci JL. Histology and histomorphometric analysis of the normal and atrophic heel fat pad. Foot Ankle Int 1995;16:254 8.

[20] Paul RG, Bailey AJ. Glycation of collagen: the basis of its central role in the late complications of aging and diabetes. Int $\mathrm{J}$ Biochem Cell Biol 1996;28:1297-310.

[21] Hardley JC, Meek KM, Malik NS. Glycation changes the charge distribution of type I collagen fibrils. Glycoconjugate J 1998; 15:835-40.

[22] Wess TJ, Wess L, Miller A, Lindsay RM, Baird JD. The in vivo glycation of diabetic tendon collagen studied by neutron diffraction. J Mol Biol 1993;230:1297-303. 